\title{
Single-Pass Polarimetric SAR Interferometry for Vessel Classification
}

\author{
Gerard Margarit, Member, IEEE, Jordi J. Mallorquí, Member, IEEE, and Xavier Fàbregas, Member, IEEE
}

\begin{abstract}
This paper presents a novel method for vessel classification based on single-pass polarimetric synthetic aperture radar (SAR) interferometry. It has been developed according to recent ship scattering studies that show that the polarimetric response of many types of vessels can be described by trihedral- and dihedral-like mechanisms. The adopted methodology is quite simple. The input interferometric data are decomposed in terms of the Pauli basis, and hence, one height image is derived for each simple mechanism. Then, the local maxima of these images are isolated, and a 3-D map of scatters is generated. The correlation of this map with the scattering distribution expected for a set of reference ships provides the final classification decision. The performance of the proposed method has been tested with the orbital SAR simulator developed at Universitat Politècnica de Catalunya. Different vessel models have been processed with a sensor configuration similar to the incoming TanDEM-X system. The analysis of diverse vessel bearings, vessel speeds, and sea states shows that the map of scatters matches reasonably the geometry of ships allowing a correct identification even for adverse environmental conditions.
\end{abstract}

Index Terms-Coherent target decomposition (CTD), polarimetric synthetic aperture radar interferometry (PolInSAR), vessel classification.

\section{INTRODUCTION}

V ESSEL monitoring is an important research area, as it can help to improve surveillance and control activities. Up until now, four main options have been considered, namely, the following: 1) Transponders located onboard the vessels provide almost perfect location and identification at real time. These devices put the autonomy of the overall system at risk, as they can be disconnected. 2) Optical imagery taking photographs of the scene. In this case, the dependence on sunlight reflection makes these sensors to be unoperatable at night or in cloudy areas. 3) Acoustic sensors that deal with the underwater noise generated by the engines of ships [1], [2]. This option needs arrays of sensors that can be damaged by ships. 4) High-frequency surface wave radar that exploits the conductivity properties of the ocean surface to detect small vessels [3]. They have been designed to work in local areas.

The lessons learnt in real scenarios show that none of these options (even the synergy of some of them) is enough

Manuscript received September 29, 2006; revised March 8, 2007. This work was supported in part by the Spanish Ministerio de Educación y Ciencia (MEC) and Fondos Europeos para el Desarrollo Regional (FEDER) funds under Project TEC2005-06863-C02-01 and in part by the Spanish Ministerio de Educación y Ciencia (MEC) Grant AP2002-2313.

The authors are with the Remote Sensing Laboratory (RSLab), Universitat Politècnica de Catalunya, 08028 Barcelona, Spain (e-mail: gerardm@ tsc.upc.edu; mallorqui@tsc.upc.edu; fabregas@tsc.upc.edu).

Color version of one or more figures in this paper are available online at http://ieeexplore.ieee.org.

Digital Object Identifier 10.1109/TGRS.2007.897437 for achieving general-purpose vessel monitoring with the demanded independence, accuracy, and temporal and spatial coverage. An alternative is synthetic aperture radar (SAR) imagery, because it can monitor large areas independently of meteorological conditions. Different projects carried out by governments and official institutions, for instance, the European IMPAST [4] and DECLIMS [5], show that SAR imagery can provide important support and can even be an alternative to classical methods.

First, works in SAR imagery have pointed out that vessel classification is not reliable with the usage of segmentation methods applied to single SAR/inverse-ISAR images [6], [7]. More resolution and/or information channels are demanded. In this sense, the analysis of polarimetric SAR (PolSAR) data via coherent target decompositions (CTDs) appears to be a reliable solution [8]. The idea is to infer geometrical features from vessel scattering by using a qualitative pixel-by-pixel procedure. In this process, CTDs are adopted because they decompose the complex scattering matrix of each pixel in terms of simple mechanisms related to canonic-like scatters. Normally, these scatters describe the scattering of some structures common in most vessels, and hence, their distribution within the signature can be useful for achieving a reasonable discrimination.

The potentialities of CTD in vessel classification have been studied in some works [9], [10]. They mainly use the so-called symmetric-scattering characterization method to interpret real vessel SAR images. The results show that polarimetry can help to isolate significant scatters that are useful for identification. However, their relation with the geometry of vessels is not still clear. In order to shed light on this issue and, extensively, on the performance of CTD for vessel classification, a scattering study that describes the polarimetric behavior of vessels for the widest observation conditions possible will be very fruitful. Preliminary works have been developed in this sense at the Universitat Politècnica de Catalunya (UPC). They are based on a numerical tool (GRECOSAR) that simulates SAR images of large vessels in simple personal computers [11], [12]. The analysis of large datasets related to diverse sensors, vessels, and environmental conditions show the following: 1) Each analyzed vessel has a particular polarimetric response in which mechanisms can be described in terms of the Pauli channels and can be associated with particular parts of the vessel structure; 2) the response of some key scatters is invariant within a cone angle around $30^{\circ}$ [11], [13]; and 3) PolSAR presents limitations for vessel classification depending on vessel characteristics, image resolution, and environmental conditions.

According to these results, a new vessel-classification method that is based on polarimetric SAR interferometry 

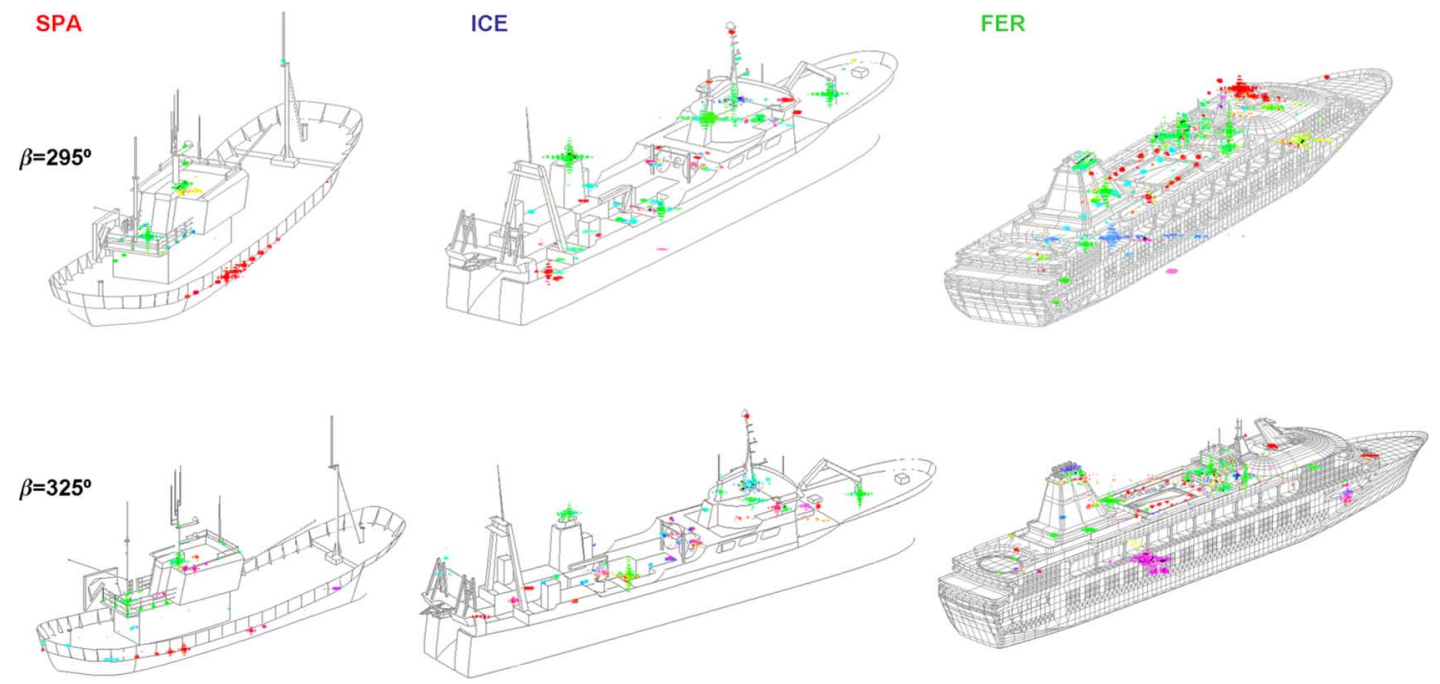

Fig. 1. Centimetric scattering maps derived from GRECOSAR for the SPA, ICE, and FER models. They have been analyzed with the Pauli theorem, and the results are impressed over a snapshot of the vessel. The following color code has been adopted: Red $\rightarrow$ trihedral, Green $\rightarrow$ dihedral $0^{\circ}$, and Blue $\rightarrow$ dihedral $45^{\circ}$.

(PolInSAR) has been proposed with the aim to overcome some of the limitations of classical PolSAR [14], [15]. It applies the Pauli theorem to the interferometric data and, then, retrieves the height of the most significant scatters. This allows to generate a 3-D map of scatters that, first, schematizes the structure of vessels and, second, allows the development of a reliable decision rule based on quantitative estimates.

This novel vessel classification method (Pauli-PolInSAR) is the main concern of this paper. It is structured as follows. In Section II, an overview of the vessel scattering study supporting the current method is provided. Details about the adopted methodology and the most important results are outlined. Section III describes the method. The focus is placed on the classification patterns and decision rule. In Section IV, the method is tested with simulated PolInSAR datasets provided by GRECOSAR for a sensor configuration similar to the future TanDEM-X [16]. The analysis of some vessel models within diverse scenarios shows that the map of scatters matches the geometry of ships in most cases. As demonstrated later, this allows us to reach a correct identification even when SAR signatures suffer from notable distortions due to the sea surface. Finally, Section V gives some guidelines about important issues that must be discussed before adapting the current method to real scenarios.

\section{Vessel ScAtTERING Study}

UPC has developed GRECOSAR, a SAR simulator of complex targets able to generate PolSAR, PolInSAR, and polarimetric inverse SAR (PolISAR) data for flexible scenarios [11]. It is based on the UPC's Graphical Electromagnetic Computing (GRECO) solver [12] that estimates in the frequency domain the radar cross section (RCS) of 3-D complex targets via high-frequency methods. Several formats are admitted, but the computer-aided design package GiD of the International Center of Numerical Methods for Engineering has been adopted [17]. The proper performance of GRECOSAR has been validated with exhaustive tests carried out for both simple and complex targets [11].

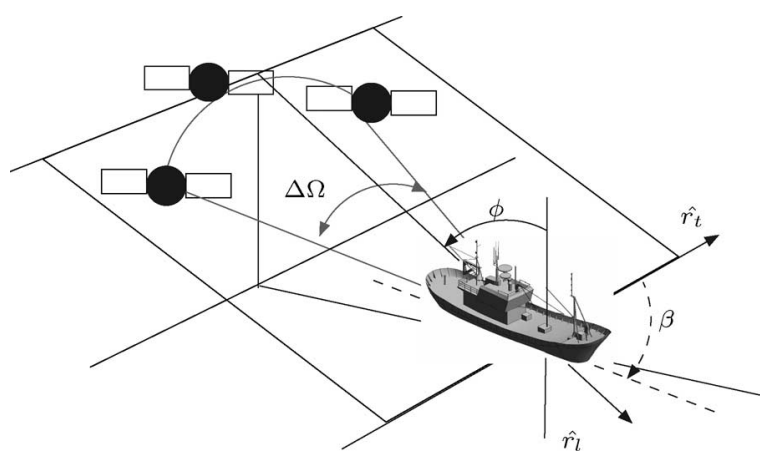

Fig. 2. Imaging geometry for the inverse SAR imagery mode of GRECOSAR.

In previous works, GRECOSAR has been used to derive a high amount of PolISAR images obtained with centimetric resolutions for different vessels and environmental conditions [11], [13]. The analysis with CTD shows the following items, namely, as follows: 1) similar polarimetric interpretation is retrieved for all CTD, and hence, any theorem is valid for the application in mind; 2) the main scattering centers can be described by trihedral/dihedral behaviors; and 3 ) the response of the vessels allows a proper target identification. It appears to be stable within a cone angle around $30^{\circ}$ (this value comes from the analysis of simulated data. When real imagery becomes available, it is expected that this value could be more restrictive).

Some snapshots of the mentioned images are presented in Fig. 1. They provide the scattering maps of three different vessels, namely, as follows: 1) a Spanish fishing vessel (SPA), $27 \mathrm{~m}$ long and $10 \mathrm{~m}$ wide, 2) an Icelandic fishing vessel (ICE), $70 \mathrm{~m}$ long and $12 \mathrm{~m}$ wide, and 3) a common passenger ferry (FER), $200 \mathrm{~m}$ long and $30 \mathrm{~m}$ wide. The adopted scenario is presented in Fig. 2, where $\beta$ defines the bearing and $\varphi=20^{\circ}$, the incidence angle. All the maps have been analyzed with the Pauli theorem, and as observed, they allow a high scatter discrimination.

In a second step, the previous experiment has been repeated with PolSAR imagery (for the same observation conditions) but 


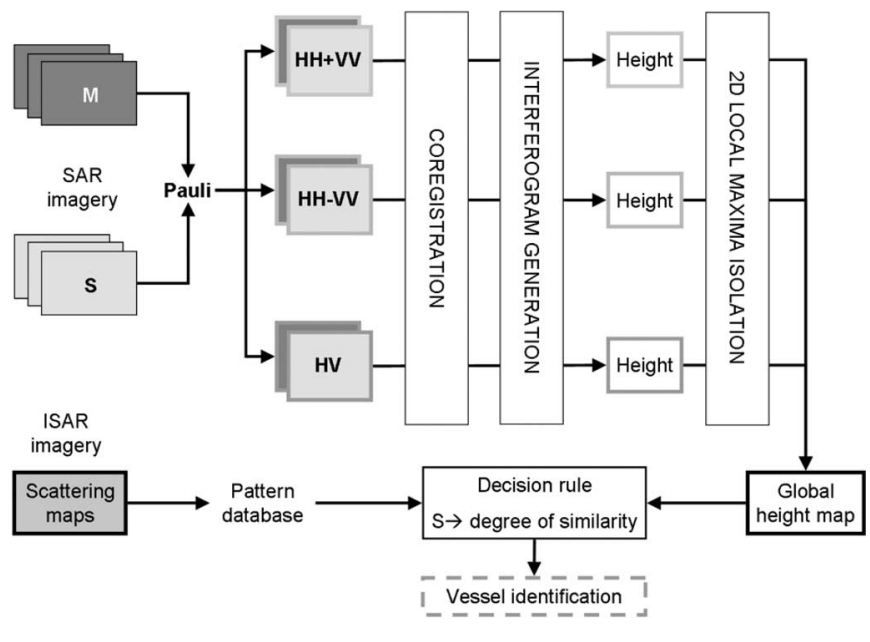

Fig. 3. Scheme of the Pauli-PolInSAR classification technique.

for resolutions ranging from 5 to $1 \mathrm{~m}$ [11]. The results have shown that CTD present some limitations for vessel classification under adverse environmental conditions and limited image resolutions. Important restrictions are that they do not retrieve quantitative estimates of geometrical features and the results can be affected by the azimuth distortions induced by the sea state [11], [18]. In this context, this paper tries to expand the information derived from CTD with single-pass interferometry. As stated by the previous scattering study, the relative height among scatters, combined with a suitable polarimetric analysis, appears to be an efficient solution for achieving an accurate 3-D scatter isolation.

It is important to note the initial character of this paper as the outlined concepts are only supported with simulated data. In despite of that, it has been shown that the adopted numerical tool provides realistic results, it does not include, for instance, the sea clutter, and consequently, validation with real data becomes mandatory once available. With the aim to advance some useful ideas, preliminary comparisons have been performed with the study in [10]. Some similarities were found. First, in that work, the key scatters used for identification behave as dipole, just the combination of a trihedral plus a dihedral. In addition, they keep their polarimetric properties for different views, as the reference hot spots observed in simulated data. In some particular situations, both datasets present some differences regarding the polarimetric mechanisms. They may be caused by the different incidence angles adopted in both cases and by the geometry of vessels.

\section{Pauli-Polinsar Method}

The scheme of the proposed classification method is illustrated in Fig. 3 [14], [15]. In the first step, the polarimetric analysis of the input data is performed via CTD. For such process, any theorem is valid because the mechanisms expected for the main scattering centers are like trihedrals and/or dihedrals, which is common in all the decomposition basis. The proposed approach uses the Pauli due to its simplicity and the fact that the orthogonality of this basis makes phase isolation easier. In this context, the evaluation of the performance of all CTD would be indeed interesting. However, it is expected that none of the different options can improve the performance achieved with the Pauli theorem.

For the $i$ th pixel with the monostatic scattering matrix

$$
S_{i}^{m}=\left[\begin{array}{ll}
\left(S_{h h}\right)_{i}^{m} & \left(S_{h v}\right)_{i}^{m} \\
\left(S_{h v}\right)_{i}^{m} & \left(S_{v v}\right)_{i}^{m}
\end{array}\right]
$$

the Pauli theorem leads to the complex Pauli vector $k_{i}^{m}$

$$
\begin{aligned}
k_{i}^{m} & =\frac{1}{\sqrt{2}}\left[\begin{array}{lll}
k_{i}^{1 \mathrm{st}, m} & k_{i}^{2 \mathrm{nd}, m} & k_{i}^{3 \mathrm{rd}, m}
\end{array}\right] \\
& =\frac{1}{\sqrt{2}}\left[\begin{array}{lll}
\left(S_{h h}+S_{v v}\right)_{i}^{m} & \left(S_{h h}-S_{v v}\right)_{i}^{m} & \left(2 S_{h v}\right)_{i}^{m}
\end{array}\right]^{\mathrm{T}}
\end{aligned}
$$

where $m$ stands for master $(m=M)$ or slave $(m=S)$ image, and $[\ldots]^{\mathrm{T}}$ denotes transpose operation. In the previous expression, $k_{i}^{1 \mathrm{st}, m}$ refers to trihedral (odd number of reflections), $k_{i}^{2 n d, m}$ refers to dihedral $0^{\circ}$ (even number of reflections), and $k_{i}^{3 \mathrm{rd}, m}$ refers to dihedral $45^{\circ}$ (antisymmetric components).

The second step applies the proper coregistration techniques in order to derive the interferogram for each channel. As the algorithm works directly with the scattering matrix, which is a first-order polarimetric descriptor, the coregistration is based on the cross correlation of the squared amplitudes of the two images in all channels. This process results on three interferometric samples for the $i$ th pixel

$$
\begin{aligned}
& I_{i}^{1 \mathrm{st}}=k_{i}^{1 \mathrm{st}, M} \cdot\left(k_{i}^{1 \mathrm{st}, S}\right)^{*} \\
& I_{i}^{2 \mathrm{nd}}=k_{i}^{2 \mathrm{nd}, M} \cdot\left(k_{i}^{2 \mathrm{nd}, S}\right)^{*} \\
& I_{i}^{3 \mathrm{rd}}=k_{i}^{3 \mathrm{rd}, M} \cdot\left(k_{i}^{3 \mathrm{rd}, S}\right)^{*}
\end{aligned}
$$

where $(\ldots)^{*}$ denotes complex conjugate. For each "Pauli interferogram," three height images $H^{1 \mathrm{st}}, H^{2 \mathrm{nd}}$, and $H^{3 \mathrm{rd}}$ are derived. In them, the local maxima that is related to a fixed dynamic range provide the relative heights of the most important scattering centers, i.e., those scatters that can lead to a proper identification. Their 3-D distribution is used to build a map of scatters that provides a reasonable representation of the structure of vessels. This map bases the decision rule of the algorithm, which is run with a pattern database that gathers the scattering information expected for a set of reference vessels. In this way, the algorithm classifies the observed ship with the pattern providing the highest similarity.

The main advantage of the current method is the retrieval of the third dimension of the space that allows, jointly with a proper polarimetric processing, a reliable vessel identification based on quantitative measurements. This improvement can be observed in Fig. 4, where simulated data is analyzed with the Pauli theorem and with the Pauli-PolInSAR method. These data are related to a target with two pairs of a trihedral and a dihedral located within two different resolution cells. These scatters have an edge length of $1 \mathrm{~m}$, and they face the sensor. The X-band system summarized in Table I has been adopted. As observed, the four scatters are isolated in both cases with their proper polarimetric behavior, but with interferometry, the available information is improved with their heights. 

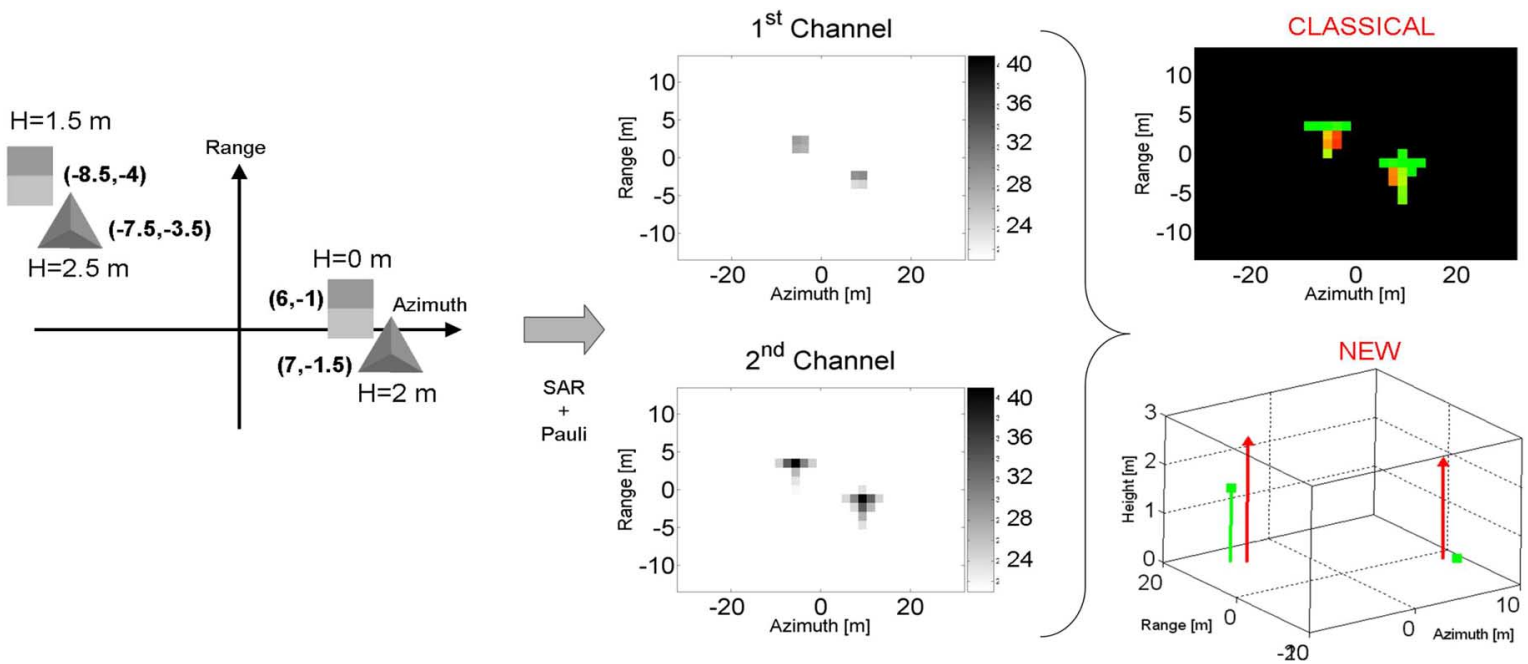

Fig. 4. Analysis results for the simulated dataset related to the array of canonics. The log-magnitude of the first and second Pauli channels are attached jointly with two colored images. The upper image shows the red/blue/green image related to the Pauli theorem, whereas the lower one shows the 3-D map of scatters derived by Pauli-PolInSAR. In both cases, the red/green color outlines the information of the first/second Pauli channels.

TABLE I

X-BAnd SEnsor Emulating TerraSAR-X

\begin{tabular}{|c|c|c|c|c|c|}
\hline$\Delta \omega_{g}[\mathrm{~km}]$ & 7 & $\mathrm{~h}[\mathrm{Km}]$ & 514 & $\varphi\left[^{\circ}\right]$ & 20 \\
\hline $\mathrm{f}[\mathrm{GHz}]$ & 9.65 & PRF $[\mathrm{Hz}]$ & 3630 & $L_{a}, L_{r}[\mathrm{~m}]$ & $4.6,1$ \\
\hline BW $[\mathrm{MHz}]$ & 135 & FS $[\mathrm{MHz}]$ & 150 & $\tau[\mu s]$ & 25 \\
\hline
\end{tabular}

The previous data show another important feature. In the case that a cell has two or three scatters and that they behave close to the mechanisms of the Pauli basis, the proposed method obtains their heights [19]. But if they share the same mechanism, the height of their center of phase is retrieved instead. Note that this methodology is a particular case of the general theory developed for the characterization of urban areas [20], [21]. In that case, the idea is to find which combination of scattering mechanisms (not necessarily orthogonal) allow to reach for each pixel the maximum of coherence and, hence, the best quality in the retrieved interferometric phase. As in the current case, it is possible to retrieve up to three different heights in a resolution cell.

\section{A. Classification Patterns}

The classification patterns used in the decision rule provide the relative height, ground location, and Pauli mechanism of a set of reference points within the structure of vessels [15]. These points are termed Persistent Polarimetric Scatters (PePS), and they present two main characteristics, namely, as follows: 1) they have the highest RCS values in the polarimetric response of vessels with a difference larger than $10 \mathrm{~dB}$ with respect to the surrounding scatters and 2) their scattering properties are preserved for a cone of angular section of around $30^{\circ}$.

PePS are identified by analyzing large datasets of scattering maps obtained for a wide observation conditions (see Fig. 1). With these images, the map height that Pauli-PolInSAR can provide is predicted, and an "expected map" for a fixed solid angle is defined. The selection of these guide scatters is performed
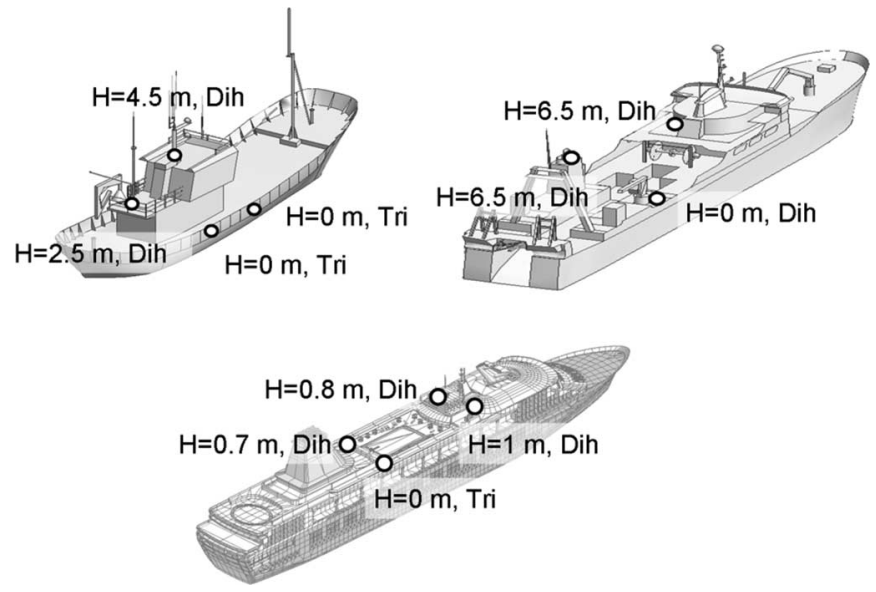

Fig. 5. Classification patterns for the vessels presented in Fig. 1. Full white circles highlight PePS locations, whereas black labels the corresponding height and Pauli mechanism ("Tri" $\rightarrow$ trihedral, "Dih" $\rightarrow$ Dihedral $0^{\circ}$ ).

according to two main considerations, namely, as follows: 1) the relative distance and height among PePS should be the maximum possible and 2) they should be placed in clean scattering areas, i.e., areas where no other scattering centers could interfere them. As commented later, such considerations are very useful to relax resolution requirements and to reduce the influence of some known distortions due to the sea environment [11], [18]. For the vessels presented in Fig. 1, the patterns illustrated in Fig. 5 are derived. They are valid for $\left(275^{\circ} \leq \beta \leq 355^{\circ}\right)$ and $\left(10^{\circ} \leq \varphi \leq 40^{\circ}\right)$.

\section{B. Decision Rule}

The correlation process between the measured signature and the pattern database is carried out in three stages. It uses a similarity parameter $0<S<1$ to establish the degree of similarity between the observed vessel and the different patterns. This parameter can be understood as a Euclidean distance between 
the locations of the main scatters isolated in the SAR image and the locations of PePS in the processed pattern. It is defined as

$$
S \doteq \frac{r}{R} \cdot\left(1-\sum_{j=1}^{4} e_{j} \cdot W_{j}\right)
$$

where $0 \geq e_{j} \geq 1$ are four different errors fixed by

$$
e_{j}=\frac{1}{r} \sum_{n=1}^{r} e_{j, n}
$$

with $j \in\{$ "azi," "ran," "hei," and "pol" $\}$ indicating the socalled azimuth $\left(e_{\text {azi }}\right)$, range $\left(e_{\text {ran }}\right)$, height $\left(e_{\text {hei }}\right)$, and polarimetric $\left(e_{\mathrm{pol}}\right)$ errors. These errors point out the mean error that is made when the value of these parameters is retrieved for all the main scatters of the measured map. In these expressions, $r$ indicates the number of PePS with a corresponding reference scatter in the measured map, and $R$ is the total number of PePS in the processed pattern. As observed later, the ratio $r / R$ plays an important role for discriminating those patterns that have no relation with the measured structure. The factors $\left(0 \geq W_{j} \geq 1\right.$ for $\left.j \in\{1, \ldots, 4\}\right)$ are weights that give different "significance" to each error in the identification process. Empirical analysis have shown that the following values provide the best overall results $\left(W_{\text {hei }}=0.35, W_{\text {pol }}=0.35, W_{\text {azi }}=0.15\right.$, and $W_{\text {ran }}=0.15$ ). They reduce the influence of azimuth and range errors that can cause severe mismatching due to the image distortions induced by sea surface [11], [18].

The expression of the azimuth error is

$$
\begin{array}{ll}
e_{\mathrm{azi}, n}=1, & \text { if } \frac{\left|P_{\mathrm{azi}, n}^{\mathrm{mea}}-P_{\mathrm{azi}, n}^{\mathrm{PePS}}\right|}{\Delta x} \geq 1 \\
e_{\mathrm{azi}, n}=0, & \text { if } \frac{\left|P_{\mathrm{azi}, n}^{\mathrm{mea}}-P_{\mathrm{azi}, n}^{\mathrm{PePS}}\right|}{\Delta x}<1
\end{array}
$$

where $P_{\mathrm{azi}, n}^{\mathrm{PePS}}$ is the azimuth location expected for the $n$th PePS of the pattern, and $P_{\mathrm{azi}, n}^{\mathrm{mea}}$ is the azimuth position of that the scattering center associated with it. Their difference is normalized to the azimuth cell dimension $\Delta x$. Similarly,

$$
\begin{array}{ll}
e_{\mathrm{ran}, n}=1, & \text { if } \frac{\left|P_{\mathrm{ran}, n}^{\mathrm{mea}}-P_{\mathrm{ran}, n}^{\mathrm{PePS}}\right|}{\Delta r} \geq 1 \\
e_{\mathrm{ran}, n}=0, & \text { if } \frac{\left|P_{\mathrm{ran}, n}^{\mathrm{mea}}-P_{\mathrm{ran}, n}^{\mathrm{PePS}}\right|}{\Delta r}<1
\end{array}
$$

where, as before, $P_{\mathrm{ran}, n}^{\mathrm{PePS}}$ is the range location expected for the $n$th PePS of the pattern, $P_{\mathrm{ran}, n}$ is the range position of that the scattering center associated with it, and $\Delta r$ is the range cell dimension. The expression for the height error is

$$
\begin{aligned}
& e_{\text {hei }, n}=1, \quad \text { if } \frac{\left|P_{\text {hei }, n}^{\text {mea }}-P_{\text {hei }, n}^{\text {PePS }}\right|}{\Delta h} \geq 1 \\
& e_{\text {hei }, n}=\frac{\left|P_{\text {hei }, n}^{\text {mea }}-P_{\text {hei }, n}^{\text {PePS }}\right|}{\Delta h}, \quad \text { if } \frac{\left|P_{\text {hei }, n}^{\text {mea }}-P_{\text {hei }, n}^{\text {PePS }}\right|}{\Delta h}<1
\end{aligned}
$$

where $P_{\mathrm{hei}, n}^{\mathrm{PePS}}$ is the height expected for the $n$th PePS of the pattern, and $P_{\text {hei }, n}$ is the height measured for that the scattering center associated with it. In this case, $\Delta h=\sigma_{h}$ is the height bias experimented by the system for a particular phase error $\left(\sigma_{\phi}\right)$ [see (9)]. Finally, the polarimetric error $e_{\mathrm{pol}, n}$ is updated to zero if the polarimetric behavior of the $n$th PePS matches the Pauli mechanism of that the scattering center associated with it. Otherwise, it is equal to one.

Based on the parameter $S$, the three-stage correlation procedure is as follows.

1) For each pattern, the distribution of the measured scattering mechanisms that better fits the PePS distribution is found. The selection of the optimal configuration does not only take into account the relative positions of such mechanisms but also their different Pauli behaviors. This means that, according to the number of PePS in the pattern and the local maxima within each Pauli channel, (4) is evaluated for all the possible permutations. Among all permutations, the one providing the highest similarity $S$ is selected. In this process, possible offsets in each error are canceled. In addition, there is a "suitability scatter" step that discards, for a fixed permutation, those measured scattering centers providing a relative height error higher than $\Delta h$ or an azimuth and range error equal to one. This modifies the value of $r$ with respect to $R$ giving sense to the ratio $r / R$ of (4).

2) Once each pattern has associated a particular distribution of local maxima in the measured signature with a specific value of $S$, the algorithm identifies the observed ship with that model having the highest similarity. In this process, the labels "PePS" and "mea" of (6)-(8) are, respectively, associated with PePS and with the measured scattering centers related to them.

3) Steps 1) and 2) are iterated for different dynamic ranges in order to isolate different combinations of local maxima. The idea is to reach the maximum similarity and/or the best discrimination among the different models. The model which pattern has been selected more times becomes the final decision of the algorithm. The final similarity value is that value providing the best discrimination among models. In the current version of the algorithm, the vessel bearing is assumed to be known according to some of the methods available in the literature [6], [22]. In the near future, it is expected to develop a vesselbearing estimator that estimates vessel bearing according to that value providing the best overall correlation between the azimuth $x$-range position of the measured scattering centers with respect to the position of the PePS of the patterns.

Note that this rule is empirical, and it has been motivated by the information that the algorithm has to deal with. It has been adopted because it allows the evaluation of the performances of the proposed method in an easy and quick way. Obviously, better decision rules may be developed in the future.

\section{Analysis With Simulated Images}

\section{A. Description of the Simulation Environment}

The performances of the Pauli-PolInSAR method have been analyzed with GRECOSAR for the three vessel models 


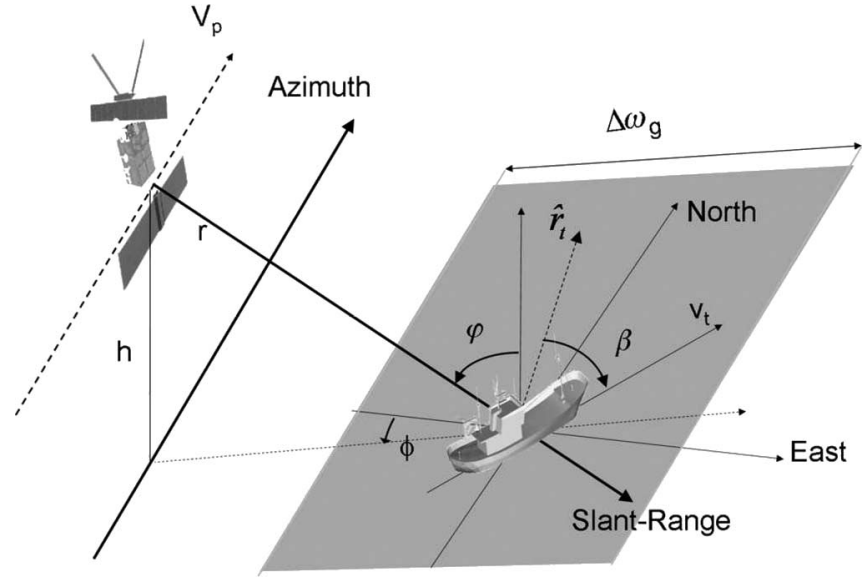

Fig. 6. Imaging geometry of GRECOSAR.

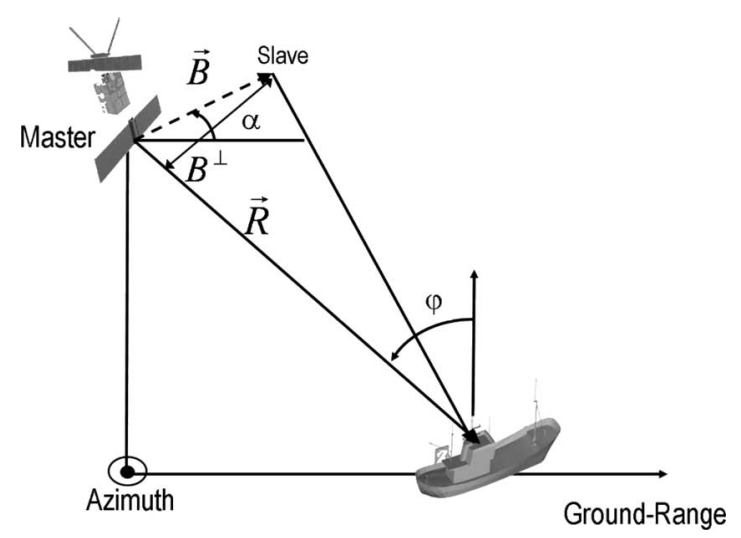

Fig. 7. Across-track interferometric geometry of GRECOSAR.

presented in Fig. 1. These models are built with parametric surfaces and meshed with a tessellation procedure that assures a discretization error lower than $3 \mathrm{~mm}$ (more than 400000 triangular facets were used). The imaging geometry of GRECOSAR is presented in Fig. 6. Besides the typical parameters related with the acquisition process and, later, data processing, the simulator can deal with some environmental parameters, namely, as follows: 1 ) vessel bearing $\beta ; 2$ ) vessel cruising speed $v_{t}$; and 3 ) vessel rotational and translational motions due to ocean waves (see [11] for further details). The adopted across-track interferometric geometry is illustrated in Fig. 7.

Table I summarizes the main parameters of the selected sensor. It is a stripmap single-pass PolInSAR system working at $\mathrm{X}$-band and based on TerraSAR-X [23]. It provides a resolution of $2.3 \mathrm{~m}$ in azimuth and $1.3 \mathrm{~m}$ in range for an antenna that is $4.6 \mathrm{~m}$ long and $1 \mathrm{~m}$ wide. The system uses the so-called pingpong measurement to maximize the effective baseline. With these values, the height sensitivity is

$$
\sigma_{h}=\frac{\lambda \sin \varphi r}{4 \pi B_{\perp}} \partial \phi=0.27 \sigma_{\phi}
$$

where $r=544 \mathrm{~km}$ is the sensor-to-target range, $B_{\perp}=30 \mathrm{~m}$ is the perpendicular baseline, and $\lambda=0.03 \mathrm{~m}$ is the operating wavelength. For the vessels processed in this paper, $\sigma_{h}$ is limited to $1 \mathrm{~m}$, and hence, $\sigma_{\phi}$ has to be $4^{\circ}$.

\section{B. Description of the Simulated Scenarios}

Four different scenarios have been processed, all summarized in Table II. In environments A and B, no sea surface is considered, whereas in environments $\mathrm{C}$ and $\mathrm{D}$, a simple model is adopted. This model considers the sea as a flat surface with a variable reflection coefficient that simulates the effect of ocean waves on the reflectivity values of the sea. This parameter is fixed by the operating frequency, the effective wave height, the elevation of the radar above the sea level, and the permittivity and conductivity of the sea. From an electromagnetic point of view, this model can compute the main contributions of sea-ship interaction to RCS allowing the derivation of accurate vessel SAR signatures [24]. This makes it possible to produce most of the scattering mechanisms observed in real data and to evaluate their influence in classification results. For the objectives of this paper, this model is enough. However, it presents a set of limitations that should be improved in future versions of GRECOSAR. They are mainly related with the absence of sea dynamics, wind conditions, and sea clutter.

In addition to the sea surface, environments $B$ and D consider pitching and rolling according to the linear terms provided in Table II. For each environment and vessel, seven bearings have been analyzed ranging from $295^{\circ}$ to $355^{\circ}$ in steps of $10^{\circ}$. This results on a total of 84 simulations, 28 for each vessel. Note that, despite that environments A and B are quite unrealistic, they are useful for isolating the effects of each environmental phenomena in vessel classification.

\section{Evaluation of the Results}

The classification results are gathered in Fig. 8 via three similar history plots. They compile the similarity values provided by the algorithm for all patterns when the 28 simulations are run for the SPA [Fig. 8(a)], ICE [Fig. 8(b)], and FER [Fig. 8(c)] ships. They are grouped in four sections according to the four sets of environmental conditions. In all cases, the red/blue/green graph highlights with the marker $+/ \circ / \times$ the similarity values obtained for the SPA/ICE/FER model. Fig. 9 shows the logmagnitude of the Pauli interferograms for simulation 1 and the SPA model. Two versions are presented for different dynamic ranges. They illustrate how the local maxima can be isolated from the whole signature and how they can be related to the PePS of the pattern.

As observed, the method is able to properly identify the correct model in most situations, even when the environmental conditions are notably adverse. In general, the overall rate of positive matches is for each model higher than $80 \%$. In addition, the algorithm achieves a good performance in three critical situations, namely, as follows: 1 ) the presence of vessel motions; 2) the influence of sea surface; and 3) the usage of vessel bearings almost parallel to the satellite track.

1) Influence of Vessel Motions: Regarding vessel motions, the notable distortions that SAR images can experiment along the azimuth direction becomes an important adverse factor for vessel classification. Analysis of real and simulated SAR data show that these phenomena can worsen the performance of methods based on image features [10], [11]. Fig. 10 presents the log-magnitudes of the Pauli interferograms retrieved for 
TABLE II

EnVironmental Conditions for the Simulations Presented in SECTION IV

\begin{tabular}{|c|c|c|c|c|c|c|c|c|c|}
\hline \multicolumn{5}{|c|}{ Environment A } & \multicolumn{5}{|c|}{ Environment $\mathrm{C}$} \\
\hline Simulation label & $\delta\left[^{\circ}\right]$ & $\dot{\delta}_{p i t c h}[\mathrm{rad} / \mathrm{s}]$ & $\dot{\delta}_{\text {roll }}[\mathrm{rad} / \mathrm{s}]$ & sea surface & Simulation label & $\delta\left[^{\circ}\right]$ & $\dot{\delta}_{\text {pitch }}[\mathrm{rad} / \mathrm{s}]$ & $\dot{\delta}_{\text {roll }[\mathrm{rad} / \mathrm{s}]}$ & sea surface \\
\hline Sim. 1 & 295 & 0 & 0 & NO & Sim. 15 & 295 & 0 & 0 & YES \\
\hline Sim. 2 & 305 & 0 & 0 & $\mathrm{NO}$ & Sim. 16 & 305 & 0 & 0 & YES \\
\hline Sim. 3 & 315 & 0 & 0 & NO & Sim. 17 & 315 & 0 & 0 & YES \\
\hline Sim. 4 & 325 & 0 & 0 & NO & Sim. 18 & 325 & 0 & 0 & YES \\
\hline Sim. 5 & 335 & 0 & 0 & NO & Sim. 19 & 335 & 0 & 0 & YES \\
\hline Sim. 6 & 345 & 0 & 0 & NO & Sim. 20 & 345 & 0 & 0 & YES \\
\hline Sim. 7 & 355 & 0 & 0 & NO & Sim. 21 & 355 & 0 & 0 & YES \\
\hline \multicolumn{5}{|c|}{ Environment B } & \multicolumn{5}{|c|}{ Environment D } \\
\hline Sim. 8 & 295 & -1.52 & -0.26 & NO & Sim. 22 & 335 & -1.52 & -0.26 & YES \\
\hline Sim. 9 & 305 & -1.43 & -0.52 & NO & Sim. 23 & 345 & -1.43 & -0.52 & YES \\
\hline Sim. 10 & 315 & -1.32 & -0.76 & NO & Sim. 24 & 355 & -1.32 & -0.76 & YES \\
\hline Sim. 11 & 325 & -1.16 & -0.98 & NO & Sim. 25 & 335 & -1.16 & -0.98 & YES \\
\hline Sim. 12 & 335 & -0.98 & -1.16 & NO & Sim. 26 & 345 & -0.98 & -1.16 & YES \\
\hline Sim. 13 & 345 & -0.76 & -1.32 & NO & Sim. 27 & 355 & -0.76 & -1.32 & YES \\
\hline Sim. 14 & 355 & -0.52 & -1.43 & NO & Sim. 28 & 355 & -0.52 & -1.43 & YES \\
\hline
\end{tabular}

simulation 8 and the SPA model. The analysis of these images in relation to those obtained for simulation 1 (the same bearing is adopted) shows that the connection between the local maxima and the PePS of the SPA pattern is not as evident as in the case of free motion. In classical methods, this phenomenon may cause important mismatches. But in the current method, it does not appreciably modify the ratio of positive matches (see motion environments B and D). Note that the high incidence adopted in these images makes pitching to cause the largest distortions. For grazing incidences, rolling appears to take more significance [25].

2) Influence of the Sea Surface: The sea surface can also modify classification results due to the new scattering processes that it can generate with the structure of vessels and that can alter the scattering information related to the imaged ships. For current simulations, the influence of the sea is not important due to the high incidence adopted and the absence of sea clutter. Future versions of the simulator should include this phenomenon as it can be an important source of noise for the interferometric phase with rough sea.

3) Influence of Extreme Bearings: The usage of polarimetry in vessel classification can also be affected by the bearing of the ship. Particularly difficult are the orientations that put the vessel almost parallel (or perpendicular) to the satellite track. In those situations, the dihedral-like mechanisms generated at the lateral sides of the cabin/hull provide a high backscattered power that can mask some of the key mechanisms required for a proper vessel identification. In this paper, this phenomenon increases the density of fails around bearings $\beta=345^{\circ}$ and $355^{\circ}$, particularly for the ICE plot.

\section{REQUIREMENTS FOR REAL SCENARIOS}

In the previous section, the analysis of simulated data have shown that the Pauli-PolInSAR method may be reliable for vessel classification. However, tests with real imagery are mandatory in order to fully validate this point. For such process, useful ideas are outlined in this section.

According to simulation results, the Pauli-PolInSAR method provides reliable results if single-pass PolInSAR sensors with recommended resolutions in the 1-3-m range are adopted, in despite that better resolutions will be always welcome. Nowadays, such resolutions are only available from some airborne sensors and, probably, from the incoming TanDEM-X orbital sensor (see Table I for guide parameters) [23].

For the application in mind, either orbital and airborne sensors are valid. The synergy of both, if possible, may be the best solution in order to find the best tradeoff between coverage and revisiting time. Note, for example, that the expected coverage for TerraSAR-X sensor in dual mode will not be larger than $15 \mathrm{~km}$ with a revisit cycle higher than one day.

Independent of the adopted sensor, it should operate at high incidence because the influence of the sea is less noticeable. Certainly, with this geometry, it is more difficult to observe strong dihedral-like mechanisms that can mask the response of vessels. Such mechanisms are more evident at medium incidences in the lateral side of the hull.

In the previous simulations, the ping-pong measurement mode is used due to restrictions on GRECOSAR. In real scenarios, the standard mode is more advisable, because the pulserepetition frequency requirements are less restrictive. However, this implies to use real baselines twice the effective one. In this sense, the value of $30 \mathrm{~m}$ previously adopted is translated to $60 \mathrm{~m}$, which can be challenging for an orbital sensor. Some tests show that effective baselines of $20 \mathrm{~m}$ may be also useful.

\section{CONCLUSION}

In this paper, a new approach for vessel classification based on single-pass PolInSAR has been presented. It infers a 3-D 


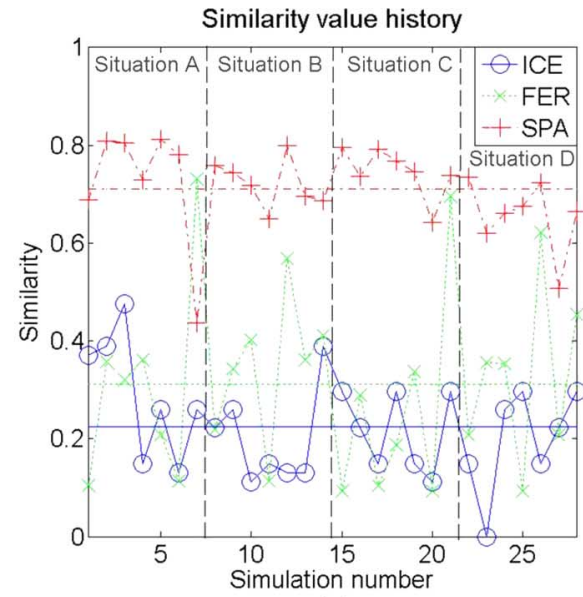

(a)

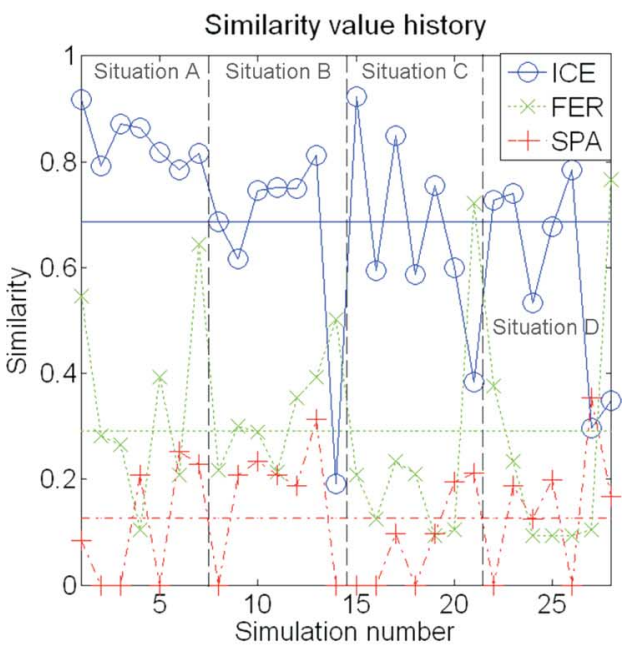

(b)

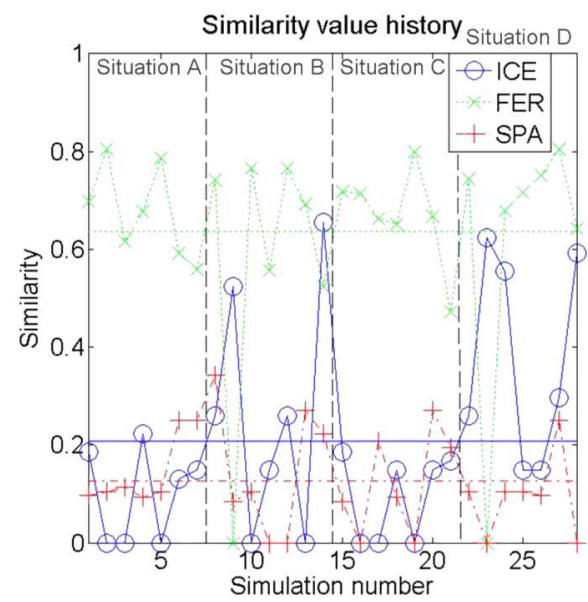

(c)

Fig. 8. Similarity history plots retrieved for simulations 1-28 when the (a) SPA, (b) ICE, and (c) FER ships are processed.

representation of the geometry of ships by combining Pauli data analysis with height-retrieval techniques. The performances of this method have been evaluated with simulated PolInSAR datasets obtained from GRECOSAR. Different scenarios for diverse environmental conditions have been analyzed for three types of ships. The results prove the good performance of the method for realistic systems, for instance, the incoming
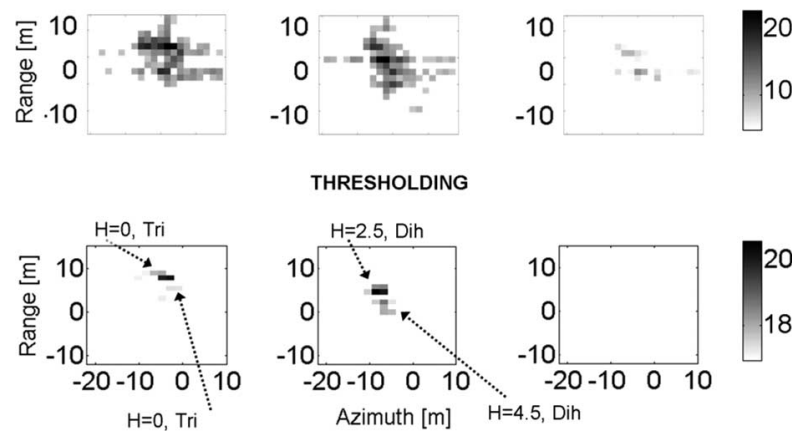

Fig. 9. Log-magnitudes of the Pauli interferograms retrieved for the SPA model in simulation 1 . The upper images are presented with a dynamic range of $20 \mathrm{~dB}$, whereas the lower ones for a dynamic range of $5 \mathrm{~dB}$.
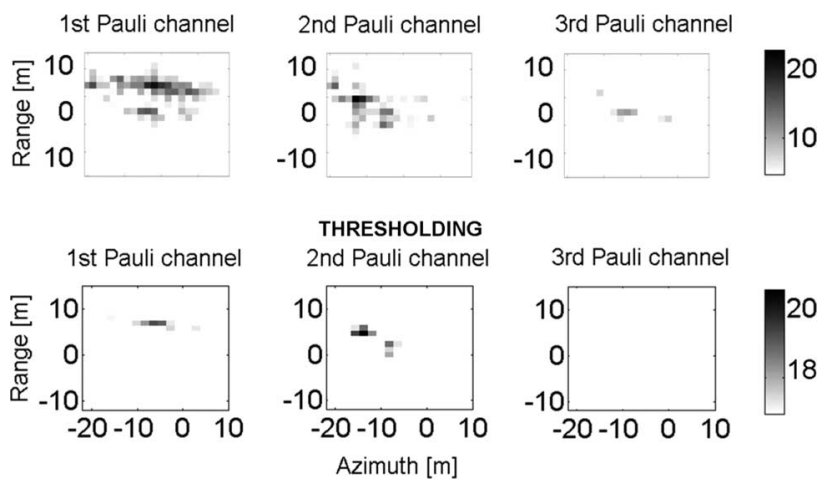

Fig. 10. Log-magnitudes of the Pauli interferograms retrieved for the SPA model in simulation 8 . The upper images are presented with a dynamic range of $20 \mathrm{~dB}$, whereas the lower ones for a dynamic range of $5 \mathrm{~dB}$.

TanDEM-X. In most cases, the processed ship has been properly identified even under adverse environmental conditions. The reason is the relative height of scatters that is less affected by the sea-induced SAR image distortions. This is an important advantage in relation to classical methods.

All the results presented in this paper are based on simulated data. In this way, the analysis of real data with reliable groundtruth is essential to validate the results presented along this paper and to identify their limitations. But before real data become available, there is a set of interesting points that have to be addressed. On the one hand, the current simulated database has to be extended in order to consider more vessels and scenarios as well as ship-material information. These data will allow to explore the limits of the proposed method and to provide more reliable estimates of the rate of positive matches. In this sense, note that the low number of patterns adopted in this paper can overestimate the results. On the other hand, sea models have to be improved with more realistic features, such as sea dynamics, wind conditions, and sea clutter. This last item can be added, for instance, by considering the sea as a dynamic (changing during the observation time) facet-based surface characterized by its dielectric properties.

Besides these items, the decision rule is another field where important research has to be done and will benefit of the larger databases. The analysis of advanced rules based on neural networks or genetic algorithms, computationally more efficient, will be very fruitful when the database of vessels becomes increased. 


\section{ACKNOWLEDGMENT}

The authors would like to thank the shipyard "Astillero Armon" for providing the blueprints of the Spanish vessel.

\section{REFERENCES}

[1] Y. Zhang, J. Zheng, H. Liu, and L. Jiao, "An efficient method of radar target classification," in Proc. Int. Conf. Radar, Oct. 2001, pp. 502-505.

[2] S. Yang, "Classification of ship-radiated signals via chaotic features," Electron. Lett., vol. 39, no. 4, pp. 395-397, Feb. 2003.

[3] T. Thayaparan and J. MacDougall, "Evaluation of ionospheric sporadic-E clutter in an arctic environment for the assessment of high-frequency surface-wave radar surveillance," IEEE Trans. Geosci. Remote Sens., vol. 43, no. 5, pp. 1180-1888, May 2005.

[4] IMPAST project web page. [Online]. Available: http://fish.jrc.cec.eu.int/ fisheries/impast/index.html

[5] DECLIMS Project Web Page. [Online]. Available: http://intelligence.jrc. cec.eu.int/marine/declims/front.html

[6] S. Musman, D. Kerr, and C. Bachmann, "Automatic recognition of ISAR ship images," IEEE Trans. Aerosp. Electron. Syst., vol. 32, no. 4, pp. 1392-1404, Oct. 1996.

[7] L. Gagnon and R. Klepko, "Hierarchical classifier design for airborne SAR images of ships," in Proc. SPIE, Sep. 1998, vol. 3371, pp. 38-49.

[8] S. R. Cloude and E. Pottier, "A review of target decompositions theorems in radar polarimetry," IEEE Trans. Geosci. Remote Sens., vol. 34, no. 2, pp. 498-518, Mar. 1996.

[9] R. Touzi and F. Charbonneau, "Characterization of target symmetric scattering using polarimetric SARs," IEEE Trans. Geosci. Remote Sens., vol. 40, no. 11, pp. 2507-2516, Nov. 2002.

[10] R. Touzi, R. K. Raney, and F. Charbonneau, "On the use of permanent symmetric scatterers for ship characterization," IEEE Trans. Geosci. Remote Sens., vol. 42, no. 10, pp. 2039-2045, Oct. 2004.

[11] G. Margarit, J. J. Mallorqui, J. M. Rius, and J. Sanz-Marcos, "On the usage of GRECOSAR, an orbital polarimetric SAR simulator of complex targets, for vessel classification studies," IEEE Trans. Geosci. Remote Sens., vol. 44, no. 12, pp. 3517-3526, Dec. 2006.

[12] J. M. Rius et al., "High frequency RCS of complex radar targets in real time," IEEE Trans. Antennas Propag., vol. 41, no. 9, pp. 1308-1319, Sep. 1993.

[13] G. Margarit, X. Fabregas, and J. J. Mallorqui, "Study of the polarimetric mechanisms on simulated vessels with SAR and ISAR imaging," in Proc. IEEE Eur. SAR Symp., EUSAR, May 2004, vol. 1, pp. 447-450.

[14] G. Margarit, J. J. Mallorqui, J. M. Rius, J. Sanz-Marcos, and X. Fabregas, "Orbital single-pass interferometry for vessel detection and classification," in Proc. FRINGE Workshop, Dec. 1-6, 2005. CD-ROM.

[15] G. Margarit, J. J. Mallorqui, and X. Fabregas, "Tri-dimensional vessel classification patterns inferred via single-pass Polarimetric SAR Interferometry," in Proc. SEASAR Workshop, Jan. 2006, pp. 1-6.

[16] M. Zink, H. Fiedler, I. Hajnsek, G. Krieger, A. Moreira, and M. Werner, "The TanDEM-X mission concept," in Proc. IGARSS, 2006, pp. 1938-1941.

[17] GiD Support Database, Barcelona, Spain: Int. Center Numerical Methods Eng. (CIMNE). [Online]. Available: http://gid.cimne.upc.es/support_ team/

[18] K. Ouchi, M. Ichara, K. Morimura, S. Kumano, and I. Takami, "Non uniform azimuth image shift observed in the Radarsat images of ships in motion," IEEE Trans. Geosci. Remote Sens., vol. 40, no. 10, pp. 2188 2195, Oct. 2002.

[19] G. Margarit, X. Fabregas, J. J. Mallorqui, and L. Pipia, "Orbital polarimetric SAR interferometry simulator of complex targets," in Proc. IGARSS, Jul. 2005, vol. 3, pp. 2015-2019.

[20] R. Zandoná, K. P. Papathanassiou, I. Hajnsek, and A. Moreira, "Polarimetric and interferometric characterization of coherent scatters in urban areas," IEEE Trans. Geosci. Remote Sens., vol. 44, no. 4, pp. 971-984, Apr. 2006.

[21] E. Colin, C. Titin-Schnaider, and W. Tabbara, "An interferometric coherence optimization method in radar polarimetry for high-resolution imagery," IEEE Trans. Geosci. Remote Sens., vol. 44, no. 1, pp. 167-175, Jan. 2006.

[22] G. Zilman, A. Zapolski, and M. Marom, "The speed and beam of a ship from its wake's SAR images," IEEE Trans. Geosci. Remote Sens., vol. 42, no. 10 , pp. 2335-2343, Oct. 2004.
[23] A. Roth and R. Werninghaus, "Status of the TerraSAR-X mission," in Proc. IGARSS, 2006, vol. 3, pp. 1918-1921.

[24] J. M. Rius, Manual of GRECO v8.6. [Online]. Available: http:// www.tsc.upc.edu/sar/pdfs/margarit_usersmanualo_.pdf

[25] K. Jamil and R. J. Burkholder, "Radar scattering from a rolling target floating on a time-evolving rough sea surface," IEEE Trans. Geosci. Remote Sens., vol. 44, no. 11, pp. 3330-3337, Nov. 2006.

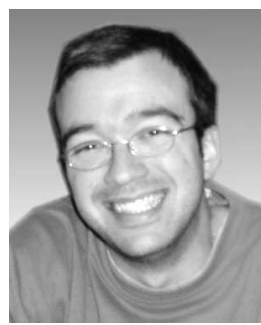

Gerard Margarit (M'05) was born in Barcelona, Spain, in 1979. He received the Diploma degree in telecommunications engineering, the Advanced Studies Diploma, and the Master MERIT Diploma degrees from the Universitat Politècnica de Catalunya, Barcelona, Spain, in 2002, 2004, and 2006, respectively, where he has been working toward the Ph.D. degree at the Remote Sensing Laboratory Department under an official Spanish Grant since 2002.

In 2004, he has hold the position of "Stagier" at the Joint Research Centre, Ispra, Italy, for a period of four months. His main research topics are 3-D SAR simulation of complex targets, polarimetric SAR interferormetry, and vessel classification.

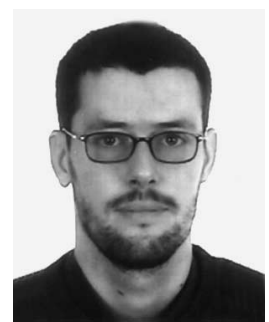

Jordi J. Mallorquí (S'93-M'96) was born in Tarragona, Spain, in 1966. He received the Ingeniero degree in telecommunications engineering and the $\mathrm{Ph} . \mathrm{D}$. degree in telecommunications engineering for his research on microwave tomography for biomedical applications from the Universitat Politècnica de Catalunya (UPC), Barcelona, Spain, in 1990 and 1995 , respectively, where in 1991, he was with the Department of Signal Theory and Communications as a Ph.D. student.

In 1993, he became an Assistant Professor, and since 1997, he has been an Associate Professor in the Telecommunications Engineering School, UPC. His teaching activity involves microwaves, radionavigation systems, and remote sensing. In 1999, he spent a sabbatical year at the Jet Propulsion Laboratory, Pasadena, CA, working on interferometric airborne SAR calibration algorithms. He is currently working on the application of SAR interferometry to terrain-deformation monitoring with orbital and airborne data, vessel detection and classification from SAR images, and 3-D electromagnetic simulation of SAR systems. He is also collaborating in the design and construction of a ground-based SAR interferometer for landslide control. Finally, he is now developing the hardware and software of a bistatic parasitic SAR for interferometric applications using ERS and ENVISAT as sensors of opportunity. He has published more than 80 papers on microwave tomography, electromagnetic numerical simulation, and SAR processing, interferometry, and differential interferometry in referred journals and international symposia.

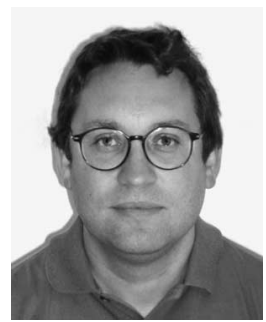

Xavier Fàbregas (M'90) received the B.S. degree in physics from Barcelona University, Barcelona, Spain, in 1988, and the Ph.D. degree in applied sciences from the Universitat Politècnica de Catalunya (UPC), Barcelona, in 1995.

Since 1996, he has been an Associate Professor with UPC. In 2001, he spent an eight-month sabbatical period at the HR Institute of the German Aerospace Agency, Oberpfaffenhofen. His current research interests include radar polarimetry, polarimetric-retrieval algorithms, and polarimetric calibration. 